\title{
Quand la présence vocale assouplit l'oral préparé : à propos des enchaînements pseudo- dialogaux entre discours radiojournalistique et citations enregistrées
}

\author{
Bertrand Verine \\ Université Paul-Valéry Montpellier 3, CNRS, Praxiling UMR 5267, France \\ bertrand.verine@univ-montp3.fr
}

\begin{abstract}
Résumé. Plusieurs recherches récentes appliquées aux médias audiovisuels ont souligné les différences de contextualisation entre, d'une part, les formes classiques de discours rapporté par la voix du journaliste et, d'autre part, les citations enregistrées. En particulier, la présentification matérielle du locuteur enchâssé via l'enregistrement restreint les possibilités d'anaphore pronominale et d'enchaînement par un connecteur argumentatif en aval de la citation, contrairement à la grande liberté qu'atteste la presse écrite après les énoncés entre guillemets. En symétrie, on montre ici que le discours d'information radiophonique actualise, avant et après les citations enregistrées, des enchaînements improbables dans le cas des discours rapportés classiques. Ainsi, en amont de l'enregistrement, il arrive que le présentateur court-circuite le travail de l'envoyé spécial en faisant mine d'être lui-même à l'initiative de l'échange avec un témoin, ce qui subvertit la frontière générique entre interview en direct et reportage en différé. Mais surtout, en aval, on rencontre des interventions évaluatives ou quasi réactives. On explique globalement ces occurrences par le fait que la matérialité de la voix du locuteur enchâssé fait ressurgir, chez le journaliste enchâssant, des réflexes dialogaux, qui lui offrent de surcroît des occasions de complicité avec les auditeurs. Le corpus est tiré des matinales de la chaîne publique France Inter de 2007 à 2017.
\end{abstract}

\begin{abstract}
When the presence of a recorded voice gives prepared speech more flexibility: would-be exchanges between radio journalists and sound-bite quotes. Several recent publications analyzing reported speech in audiovisual media have pointed out the differences in contextualization between, on the one hand, classical instances of reported speech used by journalists and, on the other, recorded quotations. It is particularly interesting to observe how, in the case of recorded quotations, the presence of a voice other than that of the journalist limits the range of anaphoras and argumentative connectors that can come after the quotation. By comparison, segments of reported speech between quotation marks in written papers give the journalist a much greater latitude to complete the sentence. However, recorded quotations also allow the journalists another kind of freedom and this is what this paper is focussing on. Before the recorded sound bite, a presenter may sometimes "borrow" the work of the special correspondent, speaking as if he himself was the initiator of the recorded exchange with an interviewed person. In doing so, he blurs the
\end{abstract}


border between two discursive genres: the live interview and the recorded report. After the recorded quotation, he also frequently makes evaluative comments, or even reacts as if he was engaged in a quasi exchange with the recorded voice. These phenomena can be explained by the presence of the recorded interviewee's voice which triggers dialogic reflexes on the journalist using the quotes. They also create opportunities for complicity with the radio audience. The corpus was selected from the morning programs of the public broadcast France Inter from 2007 to 2017.

La dialectique entre la langue parlée et sa circulation médiatique trouve un point d'impact particulièrement intéressant dans l'enchâssement de citations enregistrées au sein du discours d'information radiophonique. Là, en effet, tout est parlé au sens restreint du mot puisque, contrairement à la télévision et aux médias numériques, rien ne peut être montré du contexte spatial ni de la gestualité contribuant aux énonciations. Mais tout est également parlé au sens plein du mot puisque, par opposition à ces mêmes médias et à la presse écrite, rien ne peut être transcodé graphiquement: en dehors de quelques messages minimaux affichables sur certains récepteurs haute fidélité, les seules incrustations ou surimpressions possibles seront des sons d'ambiance, de la musique, voire des bruitages le cas échéant (Verine 2013). Cette langue parlée alterne et, le plus souvent, contraste avec l'oral préparé des journalistes ou des animateurs (à l'exception de quelques magazines qui affichent délibérément une familiarité stylisant la parole spontanée).

De manière générale, on sait que les expressions ${ }^{i}$ exogènes aux médias audiovisuels correspondent à trois types de situations d'énonciation (voir Jamet et Jannet 1999 : 90-140 notamment). Elles peuvent d'abord être produites en simultanéii par des invités, des auditeurs ou des téléspectateurs, cas qui ne nous intéressera pas ici. Elles peuvent ensuite être proférées par la voix du journaliste qui les cite selon une des procédures du discours rapporté (désormais DR) ou de la représentation du discours autre avec mention, cas auquel je me référerai seulement à titre contrastif, et qui - à quelques variantes près - est assimilable aux utilisations des guillemets et/ou des italiques dans la presse écrite (voir Authier-Revuz 1993). Elles peuvent enfin être convoquées grâce à l'enchâssement, dans la parole du journaliste, d'autres actes locutoires préalablement enregistrés, que Jamet et Jannet singularisent en les dénommant voix différées et exogènes (ibid. : 138).

Les procédures d'enchâssement de ces citations enregistrées sont, pour l'essentiel, les mêmes que dans le second cas: discours direct ( $\mathrm{X}$ a dit $\mathrm{P}$ ), modalisation autonymique d'emprunt (pour X, P), îlot textuel / discursif avec ou sans explicitation de l'autre situation d'énonciation. Cependant, une des spécificités des restitutions machiniques consiste à favoriser, aussi bien à leur amont qu'à leur aval, des enchaînements en principe caractéristiques de l'échange interactionnel, comme en (1) ${ }^{\mathrm{iii}}$ :

(1) [présentateur] alors bien sûr Franck [Ballanger] vous avez rencontré l'homm(e) de la $\mathrm{s}(\mathrm{e})$ main(e) c'est Amory Leveau il a en huit jours fait tomber cinq records du mond(e) c'est bien ça Amory cinq $\uparrow$

[A. Leveau] ouais cinq ouais / c'est bien [rire] ça fait parler d(e) la natation en France hein c'est ça e:t $p(e u) t$-être euh en dehors de France et après moi / j(e) nag(e) pas et $\mathrm{j}(\mathrm{e})$ me dis pas $\mathrm{j}(\mathrm{e})$ vais battr(e) des $\mathrm{r}(\mathrm{e})$ cords de France tout(e)s les dix s(e)cond(e)s hein / c'est $\mathrm{j}(\mathrm{e})$ nage $\mathrm{i}:(\mathrm{l}) \mathrm{s}$ tombent voilà

[présentateur] voilà je nage $\mathrm{i}(\mathrm{l}) \mathrm{s}$ tombent et voilà [intonation rieuse] ça a l'air euh [fin de l'intonation rieuse] tout simple c'était Franck Ballanger en ligne de Rijeka sur la côte adriatique en Croatie [14.12.2008].

En amont de la citation, le présentateur s'adresse, en personne et nominativement, à l'envoyé spécial (Franck vous avez rencontré l'homm(e) de la s(e)maine), mais il court- 
circuite le relais de l'intervenant annoncé en prenant à son compte l'initiative de l'échange avec l'interviewé (c'est bien ça Amory cinq $\uparrow$ ). En aval de la citation, ce même présentateur enchaîne par une volumineuse reprise littérale (je nage i(l)s tombent voilà) et l'assortit d'un commentaire sans aucun apport informatif (ça a l'air tout simple).

Après avoir rappelé les arguments déjà documentés en faveur de la distinction entre citations représentées et enregistrées (1.), je détaillerai les formes et les effets de ces enchaînements lexicaux (2.) et syntaxiques (3.). Je m'appuierai sur un corpus recueilli au fil de l'eau dans les journaux du matin de la chaîne publique France Inter depuis 2007. Je préciserai d'emblée que mes observations ont avant tout une valeur tendancielle, compte tenu de la rapidité d'évolution qu'impliquent les changements technologiques et de la forte capacité d'hybridation des genres qu'induisent aussi bien la mise en réseau des différents médias (écrits, parlés et audiovisuels) que la multimodalité croissante des sites Internet de tous les organes d'information.

\section{Spécificité des citations enregistrées dans le champ des énoncés dialogiques}

\subsection{Locuteur enchâssé}

Les recherches récentes - et assez peu nombreuses - sur le DR dans les médias audiovisuels soulignent avant tout les points communs entre les citations enregistrées et celles que profèrent les journalistes : mode d'introduction et d'imputation (voir Munchow 2004), sélection des fragments cités (voir Jamet et Jannet 1999 ; Labeau 2007), fonction d'attestation et de reproduction (voir Prost 2005), notamment. Je m'efforce au contraire d'observer l'impact sur les journalistes et sur leur auditoire du fait que les citations enregistrées ajoutent à l'hétérogénéité énonciative, commune à toutes les formes dialogiques, une caractéristique inédite dans l'histoire du langage humain : l'hétérogénéité sonore (voire iconique) qu'impliquent leur profération par le locuteur cité et les éléments paraverbaux, coverbaux ou contextuels plus ou moins nombreux qui les accompagnent. Les voix du dialogisme (Bakhtine 1984) ne doivent donc plus, en l'occurrence, être entendues de manière figurée, mais littéralement et dans tous les sens.

De fait, la citation enregistrée déroge à l'observation, posée par Bres et Verine (2002: 166), selon quoi, dans toutes les formes dialogiques, «[la] possibilité d'effacement distingue le dédoublement de l'instance du locuteur de celui de l'instance de l'énonciateur ». Ces auteurs soulignent que, s'il y a toujours dédoublement entre deux instances déictiques et modales (un énonciateur enchâssant et un énonciateur enchâssé), (a) l'instance de production matérielle qu'est le locuteur enchâssé se manifeste beaucoup plus rarement, en particulier à l'écrit, (b) qu'elle n'apparaît que sous la forme de traces, et (c) que ces traces peuvent seulement être représentées par la profération ou la graphie du locuteur enchâssant.

La spécificité des citations instrumentées par la technologie réside au contraire en ceci qu'elles donnent à entendre (et/ou à voir) la matérialité même de la locution enchâssée en l'absence de toute interactivité et en rendant possibles de nombreuses manipulations de la part du locuteur enchâssant. J'ai montré ([] Verine 2013) que les opérations de décontextualisation/ recontextualisation propres à cet enchâssement instrumenté sont particulièrement audible dans les cas où le journaliste insère dans sa parole un îlot enregistré syntaxiquement et sémantiquement non autonome. En retour, nous observerons (infra 3.1) que la hiérarchisation des contextes peut être masquée dans certains cas où le journaliste enchâsse un enregistrement autonome en imitant les procédures de l'enchaînement dialogal, obligeant l'auditeur à un surcroît d'attention pour ne pas confondre l'alternance sonore des voix avec celle de tours de parole construisant un 
échange interactionnel. Ces phénomènes peuvent affecter aussi bien les citations extraites d'interviews préalables que celles provenant d'énoncés enregistrés sur le vif, dont les actes d'énonciation ont pu constituer en soi un événement (assemblées politiques notamment) ou accompagner l'événement (échanges dans le feu de l'action).

\subsection{Impact en retour sur le locuteur enchâssant}

J'ai commencé à montrer (Verine 2009 et 2013) que la présence de l'enregistrement agit en retour sur la profération et sur la formulation du discours des journalistes. D'une part, certains enchâssements de citations enregistrées ne vont pas sans risque d'aléas sonores comme la réduplication segmentale, que j'explique par le fait que le journaliste enchâssant anticipe si intensément sur la cohésion en aval du discours qu'il ne parvient pas à interrompre son dire sur le premier mot de la citation. Un autre aléa possible est la divergence entre les désignations en non-personne actualisées par le journaliste et les coordonnées personnelles et spatiotemporelles indexées sur le je de la source enregistrée, la radio ne disposant pas d'un équivalent des crochets parfois utilisés à l'écrit pour signaler les transpositions nécessaires à leur mise en cohérence. Plus généralement, il semble que, jusqu'à présent, la restitution machinique de la locution enchâssée restreigne les possibilités d'anaphore pronominale et d'enchaînement par certains connecteurs argumentatifs en aval de la citation, contrairement à la grande liberté qu'atteste la presse écrite (cf. infra 3.2).

Cet ensemble d'éléments m'a conduit à poser, sous l'unité en langue de chaque forme de discours rapporté avec mention, la nécessité pour l'analyse du discours d'articuler citation représentée par la voix du locuteur enchâssant et citation convoquée grâce à l'enregistrement. Je voudrais ici affiner l'inventaire des différences entre ces deux modes de citation en montrant que le discours d'information radiophonique actualise des formes inattendues d'enchaînement entre les énoncés des journalistes et les voix enregistrées qu'ils enchâssent.

\section{Surmarquage des enchâssements par des enchaînements lexicaux et/ou des segments évaluatifs}

\subsection{Annonces et reprises lexicales}

On observe d'abord des endophores fidèles très rapprochées qui n'attirent pas l'attention en écoute spontanée, alors qu'elles apparaîtraient comme marquées si la citation était représentée. Sous (2) et (3), les réécritures indexées a et b s'efforcent de montrer que, dans les occurrences exactement identiques et immédiatement contiguës, de telles répétitions pourraient être reçues, selon les contextes, soit comme des anadiploses volontaires, soit comme des ratages d'actualisation (voir Verine 2013), aussi bien à l'écrit que si le radiojournaliste proférait lui-même le discours enchâssé :

(2) [journaliste] autre saisie précieuse selon la procureur Marie-Suzann(e) Le Quéau deux pistolets automatiqu(e)s 7-65 et surtout un(e) douille

[MS Le Quéau] un(e) douille de 7-65 dont l'examen a d'or(e)s et déjà démontré qu'ell(e) prov(e)nait de l'arme utilisée pour commettre les assassinats [16.04.2012];

? et surtout, selon la procureur, une douille : « une douille de 7-65 [...] utilisée pour commettre les assassinats »; et surtout, selon la procureur, « une douille de 7-65 [...] utilisée pour commettre les assassinats $»$; 
(3) [B. Thibault] maint(e)nant tout va dépendre de la réaction des salariés

[journaliste] salariés du public et du privé app(e)lés à manifester jeudi prochain [17.06.2010] ;

? selon B. Thibault, « tout va dépendre de la réaction des salariés », salariés du public et du privé appelés à manifester jeudi prochain ; selon B. Thibault, «tout va dépendre de la réaction des salariés » appelés à manifester jeudi prochain dans le public et dans le privé.

Assez rares entre l'amont et l'attaque de la citation enregistrée, elles sont beaucoup plus fréquentes entre sa chute et l'aval de l'énoncé hôte, notamment quand elles présentent d'infimes variations et/ou de légers décalages syntagmatiques comme dans les extraits (4) à (7) :

(4) [interviewé] oui donc euh l'audience est reportée au 12 mars

[journaliste] et d'ici le 12 mars un(e) réunion entre les associations les SDF et les pouvoirs publics devrait décider de l'av(e)nir des habitants de ce local [26.02.2010];

(5) [C. Marquez] la ministre s'est engagée sur l'ensemble des points et euh nous serons bien entendu attentifs à leur mise en place

[journaliste] attentifs notamment en septembre lorsque le projet de loi de finance 2010 sera présenté [12.05.2009];

(6) [interviewée] attendr(e) deux heur(e)s pour avoir un(e) réservation c'est vraiment pénibl(e) quoi

[journaliste] pénible / et trop fréquent pour Thomas qui arriv(e) de Bordeaux [12.01.2009];

(7) [interviewé] [le médecin] c'est un(e) personne oui central(e) de la vie rurale [journaliste] centrale et pourtant de plus en plus rare [13.05.2009].

Chacune de ces répétitions pourrait être purement et simplement supprimée comme en (2b) et (3b), ou remplacée par un synonyme tel que [pièce à conviction] en (2), [personnels] en (3), par l'anaphore adverbiale (d'ici là] en (4), etc. En première approximation, tous ces exemples peuvent s'interpréter comme renforçant la cohésion textuelle au moment même où l'enregistrement inscrit l'hétérogénéité élocutive / énonciative la plus marquée. Il s'y ajoute un effet de mise en saillance particulièrement économique, aussi bien en terme de recherche lexicale que par rapport au coût qu'aurait la production d'une nouvelle phrase complète telle que [les personnels du public et du privé sont d'ailleurs appelés à manifester] en (3), ou [vigilants les syndicalistes le seront notamment en septembre] en (5). Dans certains cotextes, la reprise permet également d'articuler à la voix de l'interviewé celle d'un second témoin en (6) ou le point de vue surplombant du journaliste en (7) : sa suppression rendrait beaucoup moins accessible l'interprétation que l'attente en gare est à la fois pénible et beaucoup trop fréquente, ou que le médecin de campagne est une personne à la fois centrale et de plus en plus rare. Nous allons constater infra que d'autres cotextes peuvent rendre cette articulation divergente.

\section{2 Évaluations}

Dans les interactions spontanées, l'un des rôles des enchaînements lexicaux est de s'arrêter sur les mots de l'interlocuteur pour en évaluer appréciativement ou dépréciativement le ton, le registre ou la pertinence (voir notamment Kerbrat-Orecchioni 1990 : 187-189 et 226- 
227). Or il est frappant de les retrouver dans un emploi assez voisin en aval de certaines citations enregistrées. Soulignons la singularité de tels segments métadiscursifs dans les articles de genre reportage ou enquête d'où provient le corpus. D'une part, leur absence d'apport informatif les distingue radicalement des séquences ou des articles ayant pour visée explicite de commenter une déclaration officielle, d'analyser un programme électoral, d'expliquer un changement terminologique, etc. D'autre part, en raison de leur forte teneur subjective, ils constituent des dérogations manifestes à l'idéal de neutralité, particulièrement revendiqué sur la chaîne publique France Inter. De fait, ils ne communiquent rien d'autre à l'auditoire que les sentiments du journaliste (ou de la rédaction), qu'il s'agisse d'admiration en (8) et (9), d'amusement en (10) et (11), ou de scepticisme en (12) et (13) $)^{\text {iv }}$ :

(8) [M. Marceau] un un gest(e) par exemple qui balbutie c'est comme un mot qui trébuche enfin on peut / bégayer en $\mathrm{f}(\mathrm{ai})$ sant un geste

[journaliste] on peut bégayer en f(ai)sant un gest(e) parol(e) de Marcel marceau ${ }^{\circ} \mathrm{h}$ bonn(e) journée [24.09.2007, date du décès du mime] ;

(9) [A. Leveau] j(e) me dis pas j(e) vais battr(e) des r(e)cords de France tout(e)s les dix s(e)cond(e)s hein / c'est j(e) nage i:(l)s tombent voilà

[journaliste] voilà je nage i(l)s tombent et voilà [intonation rieuse] ça a l'air euh [fin de l'intonation rieuse] tout simple c'était Franck Ballanger en ligne de Rijeka sur la côte adriatique en Croatie [14.12.2008];

(10) [É. Gerets] on est pas morts on est drôl(e)ment vivants

[journaliste] voilà drôl(e)ment vivants l'explosion $\mathrm{d}(\mathrm{e})$ joie d'Éric Gerets l'entraîneur marseillais [16.03.2009];

(11) [N.Sarkozy] des répons(e)s qu'on va r(e)garder avec cett(e) méprisance

[journaliste] voilà la méprisance après la bravitude [28.03.2012] ;

(12) [N.Sarkozy] que personn(e) ne compt(e) sur l'impunité bon courage et bon travail

[journaliste] voilà bon courage et bon travail message adressé donc aux personnels d'éducation et aux policiers [29.05.2009];

(13) [journaliste] un candidat dont le mot d'ordre ce matin était très clair

[F. Hollande] j'assume

[journaliste] voilà François Holland(e) dit assumer [11.01.2012].

Il va de soi qu'en termes interactionnels ces segments ne fonctionnent pas comme les interventions évaluatives du dialogue, puisqu'ils ne s'adressent pas au locuteur enchâssé, mais prennent à témoin l'auditoire du journal. Outre leur parenté sémantique, les uns partagent cependant avec les autres au moins trois ressemblances formelles. En premier lieu, ils interviennent presque toujours en clôture terminale (d'article, de rubrique ou de journal), comme l'indiquent le souhait bonne journée en (8) ou le pied de reportage c'était en (9). En second lieu, on y entend les marques d'une oralité plus spontanée que dans le reste du discours enchâssant, à l'instar de l'amuïssement du $l$ de $i(l)_{s}$ et de l'intonation rieuse sur ça a l'air en (9]. Enfin, leur trait de spontanéité le plus constant est le recours quasi systématique à voilà en fonction de marqueur discursif. Dans les discours représentés en interaction vive, Branca-Rosoff et Verine (2012 : 210) ont montré que voilà constitue « une marque de transition articulant dialogique (la relation interdiscursive entre l'enquêtée et ses personnages) et dialogal (la relation interlocutive entre l'enquêtrice et l'enquêtée) ». 
Après les citations enregistrées des médias audiovisuels, la transition marquée par voilà articule le dialogisme interdiscursif (arrêt du journaliste citant sur le propos du locuteur cité) avec la relation dialogale (balisage d'une zone de partage des sentiments entre le journaliste et l'auditoire) ou, le cas échéant, avec le dialogisme interlocutif (anticipation par le journaliste de l'évaluation de l'auditoire). En 2017, ce type de fonctionnement est devenu une des caractéristiques du nouveau style rédactionnel de France Info, autre chaîne du service public de la radio française.

\section{Structuration des enchâssements en pseudo-échanges}

\subsection{Pseudo-initiatives en amont}

Les chaînes publiques françaises sont presque toujours parvenues à conjurer ce piège, dans lequel sont tombées nombre de radios privées, notamment $R M C$ Info, dont il constituait une signature autour de 2010. Dans cette période et sur cette antenne, on entendait très régulièrement le présentateur du journal introduire par une question en vous et une forme nominale d'adresse des propos d'autres locuteurs dont le fond sonore révélait qu'ils n'étaient proférés ni en studio ni au téléphone, mais qu'ils constituaient des citations enregistrées : cela revenait, donc, dans le cas le moins mauvais, à fausser les coordonnées énonciatives, mais plus souvent à transformer des reportages ou de véritables évènements de parole en pseudo-interviews. Or, en décloisonnant les situations d'énonciation, en brouillant ou en gommant leur marquage, un tel dispositif complique l'activité d'interprétation de l'auditoire, contrevient à l'engagement d'exactitude des journalistes et sape, si peu que ce soit, le contrat de communication propre à l'information médiatique en y introduisant une part de truquage. Les exemples (14) et (15) montrent qu'il est très facile d'éviter cet écueil, puisqu'il suffit que le présentateur transpose la question au discours indirect libre, donc ici en non-personne, pour que la dualité des situations d'énonciation reste audible. L'ambiguïté des extraits (16) et (17) ou l'incohérence de l'exemple (18) soulignent cependant que la grande maniabilité des enregistrements numériques soumet en permanence les journalistes à la tentation du pseudo-dialogue avec leurs sources :

(14) [journaliste] au final comment l'ancien président a-t-il trouvé ce rendez-vous $\uparrow$

[J. Chirac] excellent avec des collaborateurs que j'ai toujours beaucoup d(e) plaisir à retrouver [14.05.2008] ;

(15) [journaliste] et les vacanciers / skient-ils comme avant $\uparrow$

[interviewé] oui mais avec euh quand même un p€tit nœud à l'estomac [...]

[14.02.2017];

(16) [journaliste] alors première impression $\uparrow$ François Chérec $\uparrow$

[F. Chérec] c'est la premièr(e) fois qu'avant de prendre un(e) fonction

officiell(e)ment un président $\mathrm{d}(\mathrm{e})$ la République organis(e) ces rencontreslà [14.05.2007] ;

(17) [journaliste] Franck Azéma est l'entraîneur clermontois satisfait euh de cett(e) fin d'année $\uparrow$

[F. Azéma] le mieux ç'aurait été d'êtr(e) premiers [21.12.2014];

(18) [journaliste] alors bien sûr Franck [Ballanger] vous avez rencontré l'homm(e) de la s(e)main(e) c'est Amory Leveau il a en huit jours fait tomber cinq records du mond(e) c'est bien ça Amory cinq $\uparrow$ 
[A. Leveau] ouais cinq ouais [14.12.2008].

En (16) et (17), les présentateurs affectent leurs énoncés enchâssants d'une intonation interrogative très audible, difficilement compatible avec l'introduction d'une citation enregistrée, alors qu'ils pourraient être proférés comme des assertions sans rien changer à leur structure. En (18), l'annonce endophorique cinq $\uparrow$ se combine au maintien dans la citation enregistrée du marqueur dialogal ouais pour imiter presque parfaitement un échange interactionnel, la confusion n'étant évitée que grâce à la différence des contextes sonores et à la contradiction entre les deux prénoms en adresse Franck (l'intervieweur effectif muet) vs Amory (l'intervenant enregistré).

\subsection{Pseudo-réactions en aval}

Les exemples (15) et (18) rappellent que oui, non, si et leurs variantes) sont le plus souvent des marqueurs dialogaux d'intervention réactive confirmant ou infirmant l'initiative d'un interlocuteur - peut-être et ses substituts permettant d'en suspendre la validation. Ces motsphrases ne sont donc pas prototypiquement attendus dans le discours d'information des journalistes, dont le rôle prédéterminé est d'être à l'initiative. J'ai montré (Verine 2005) qu'un locuteur enchâssant peut en faire un emploi dialogique pour valider, contredire ou mettre en débat le contenu d'une citation qu'il profère, mais je faisais l'hypothèse (Verine 2009, rédigé en 2007) que ce fonctionnement était difficilement transposable après les citations enregistrées, en raison du surmarquage de l'enchâssement par la cohabitation sonore de deux contextes d'énonciation. Réciproquement, Bres et Dufour (2014) attestent que de tels emplois apparaissent dans les titres de presse écrite, y compris en l'absence de toute autre marque du processus citationnel, ce qu'ils expliquent par le double fait que les nouveaux médias tendent à mêler d'oralité l'écriture journalistique et que cet emprunt à la textualité dialogale donne plus de force à la prise de position du scripteur enchâssant. Les extraits (19) à (23) prouvent que ces adverbes modaux sont désormais en usage après les enregistrements enchâssés :

(19) [interv. 1] la direction a pensé que c(e) que nous portions comme revendications en term(e) de ${ }^{\circ} \mathrm{h}$ de gestion au quotidien elle a ris ça par-dessus la jambe et ell(e) nous a provoqués jusque: ${ }^{\circ} \mathrm{h}$ dans la situation où on $\mathrm{s}(\mathrm{e})$ trouve aujourd'hui

[journaliste] oui car face à cette forte augmentation du nombre de passagers sur la ligne A la RATP a mis en place un plan régularité qu'il faut respecter coût(e) que coût(e) selon Josett(e) Théophile ${ }^{\circ} \mathrm{h}$ directric(e) générale adjointe à l'innovation sociale

[interv. 2] y’a pas d(e) pression y’a un plan régularité [...] [08.06.2009];

(20) [Thierry] [journaliste]

(21) [interv. 1]

[journaliste]

[interv. 2] ce s(e)rait pas compliqué hein

pas compliqué et ça chang(e)rait la vie des non-voyants et de Thierry plus habitué à voir avec ses oreill(e)s qu'avec ses yeux [...] [...] [08.09.2016] ;

c'est la premièr(e) fois que $\mathrm{j}(\mathrm{e})$ remont(e) les Champs sans qu'y ait un(e) seul(e) voiture et c'est assez agréable

oui mais Nathalie n'est pas loin de penser 1(e) contraire en ce 27 juillet ell(e) n'est pas à la fête

on peut pas fair(e) les Champs en vélib c'est trop dang(e)reux donc je pensais qu(e) ça montait mais là c'est vraiment dur [27.07.2008] ; 
(22) [interv.]

[journaliste]

[interv.]

(23) [interv.]

[journaliste] y'a pas eu la moindre égrattignur(e) la moindre blessure y'a pas

$\mathrm{d}(\mathrm{e})$ sang dans cette affaire

peut-être mais beaucoup d(e) violence en ce 12 mars 2003

comme en témoigne ${ }^{\circ} \mathrm{h}$ ce surveillant présent au moment $\mathrm{d}(\mathrm{e})$

l'attaque

j'ai entendu le bruit de de voitur(e)s de port(e)s qui claquent et j'ai vu euh trois hommes encagoulés [...] [02.10.2008];

c'est pour ça qu'on d(e)mande un moratoire alors app(e)lons-le moratoire app(e)lons-le gel ${ }^{\circ} \mathrm{h}$ avant de dév(e)lopper de nouveaux OGM dont personn(e) ne veut en plus

si la FNSEA et les professionnels des semences en veulent les chercheurs aussi qui estim(e)nt que leurs recherch(e)s doiv(e)nt passer de

l'éprouvette au champ pour être correctement évaluées [28.09.2007].

On observe les principales nuances de la gamme des prises de position possibles par rapport à une assertion antérieure : confirmation renforcée par une justification (oui car) en (19), validation d'une assertion négative prolongée par une affirmation (pas compliqué et) en (20), confirmation relativisée par une adversation (oui mais) en (21), mise en débat au profit d'une proposition plus pertinente (peut-être mais) en (22), franche réfutation (portant sur une assertion négative, d'où l'emploi de $s i$ ) en (23). Il est vrai que je ne dispose pas encore d'occurrences d'enchaînement par non, ce qu'on peut expliquer par le caractère non préféré (voir Levinson 1983 : 335) de la réfutation frontale après une affirmation : or, dans les articles de genre reportage ou enquête, les interviewés sont prototypiquement convoqués pour asserter leur point de vue (non pour poser des questions) et le journaliste a pour visée première d'orchestrer la description d'un phénomène (non de pondérer entre eux des arguments ${ }^{v}$ ) ; cela passe, dans la très grande majorité des cas, par la sélection de citations dont le contenu explicite n'est pas radicalement contestable.

Comment, dès lors, expliquer la mise en débat de (22) et la réfutation de (23) ? Par le fait que les adverbes modaux contribuent dans tous ces cas à articuler la citation enregistrée à un autre point de vue, également porté par une voix enregistrée en (19), (21) et (22), ou représenté par l'empathie du journaliste avec les non-voyants en (20) et par le discours rapporté indirect (qui estiment que) en (23). J'affinerai donc mon hypothèse antérieure en disant que les radiojournalistes ont intégré la possibilité d'enchaîner sur une citation enregistrée par les marqueurs dialogiques oui, peut-être, si ou non, mais ils la restreignent pour le moment à l'orchestration de différents points de vue enchâssés, parce que de tels enchaînements rendent extrêmement audible leur prise de position par rapport à leurs sources. Soulignons que, même dans le cas apparemment plus consensuel de (19), le journaliste enchâssant hiérarchise bel et bien les énoncés enchâssés, en adhérant au discours syndical de l'interviewé 1 et en le justifiant par le discours patronal de l'interviewée 2, représenté comme intransigeant. Je signale enfin comme une curiosité l'extrait suivant:

(24) [interv. 1] franch(e)ment le front républicain c'est 1(e) f- c'est 1(e) front républicain pour sauver leurs places / Monsieur / alors $\uparrow$

[journaliste] [interv. 2] on hésite

Le journaliste ayant choisi d'inclure dans la citation l'initiative alors $\uparrow$ que l'interviewé lui adresse explicitement par l'apostrophe monsieur, la cohésion textuelle laisse attendre qu'il réagisse, ce qui contreviendrait au contrat générique de neutralité ; il résout la difficulté grâce à la proposition on hésite, où le prépersonnel on peut l'impliquer, mais où le 
choix du verbe hésiter permet de ne pas révéler son opinion et d'introduire les réactions d'autres interviewés.

\section{Conclusion}

Les quatre catégories de phénomènes présentées ici me paraissent confirmer la spécificité de la citation enregistrée dans le champ du discours rapporté, et l'intérêt qu'il y aurait à entreprendre une enquête de grande ampleur comparant leur traitement par différents médias francophones et dans d'autres langues, afin de savoir si les faits relevés constituent de simples accidents ou une tendance lourde justiciable d'hypothèses explicatives. Tous prouvent l'impact de la présence vocale du locuteur enchâssé sur le discours d'information radiophonique, la matérialité de la voix enregistrée faisant ressurgir, chez le journaliste citant, des réflexes dialogaux. Mais il semble, en l'état actuel des usages et de la recherche, qu'on doive distinguer trois cas de figure. D'une part, l'abondance des annonces ou des reprises lexicales (supra 2.1) manifeste des possibilités et des contraintes stylistiques nouvelles en termes de cohésion thématique / textuelle et de gestion du temps de rédaction, puis de profération du discours d'information. D'autre part, la formulation par les journalistes d'évaluations (supra 2.2) ou de pseudo-initiatives (supra 3.1) relève plutôt de la contingence, non sans effets sur le cadrage des genres du discours, voire sur le pacte de communication journalistique, qu'on les interprète comme des suspensions momentanées de la rigueur professionnelle ou comme des jeux délibérés avec l'auditoire. Enfin, la fréquence relative des pseudo-réactions (supra 3.2) et leur co-occurrence actuelle avec le passage d'une source d'information à une autre rend plus fondamentalement audible l'articulation systémique entre le dialogue externe de deux interactants et le dialogisme interne de deux énonciations au sein du même énoncé.

\section{Références bibliographiques}

Authier-Revuz, J. (1993). Repères dans le champ du discours rapporté (II). L'Information grammaticale, 56, 10-15.

Bakhtine, M. (1984). Esthétique de la création verbale. Paris : Gallimard.

Branca-Rosoff, S. et Verine, B. (2012). Le discours direct et ses limites : l'asymétrie de la frontière gauche et de la frontière droite. Branca-Rosoff, S. et al. (éd.). L'Hétérogène à l'œuvre dans la langue et le discours, Limoges : Lambert-Lucas, 199-214.

Bres, J. et Dufour, F. (2014). Du fonctionnement dialogique monologal des marqueurs dialogaux oui, non, si. Arena romanistica, 14, 56-70.

Bres, J. et Verine, B. (2002). Le bruissement des voix dans le discours : dialogisme et discours rapporté. Faits de langues, 19, 159-169.

Jamet, C. et Jannet, A.-M. (1999). La Mise en scène de l'information Paris : L'Harmattan.

Kerbrat-Orecchioni, C. (1990). Les Interactions verbales I. Paris : Armand Colin.

Labeau, E. (2007). De l'objectif au subjectif : le rapport du discours à la télévision. Broth, M. et al. (éd.). Le Français parlé des médias. Stockholm : Acta Universitatis Stockholmiensis, 365-382.

Levinson, S. C. (1983). Pragmatics. Cambridge: Cambridge University Press.

Münchow von, P. (2004). Les Journaux télévisés en France et en Allemagne. Paris : Presses Sorbonne Nouvelle.

Prost de, S. (2005). Journal télévisé et discours rapporté : une approche du discours convoqué. López Muñoz, J. M. et aL. (éd.). Dans la jungle des discours. Genres de discours et discours rapporté. Cadix : Servicio de Publicaciones de la Universidad, 413-422.

Verine, B. (2005). Dialogisme interdiscursif et interlocutif du discours rapporté : jeux sur les frontières à l'oral. Bres, J. et al. (éd.). Dialogisme et polyphonie. Approches linguistiques. Louvain-la-Neuve : De Boeck Duculot, 187-200.

Verine, B. (2009). Une variante contextuelle du discours rapporté avec mention: le discours convoqué dans le journal et l'enquête radiophoniques. Havu, E. et al. (éd.). La Langue en contexte. Helsinki : Société Néophilologique, 349-370. 
Verine, B. (2013). Une procédure déjà passée de mode et toujours d'avenir : l'îlot enregistré dans le discours d'information audiovisuelle. Cahiers de praxématique, 61, en ligne.

ii Je ne ferai que quelques allusions au travail journalistique de reformulation des contenus avec lequel alternent les citations exogènes.

ii J'évite à dessein l'expression consacrée « en direct », pour ne pas créer d'ambiguïté avec le discours direct au sens de discours rapporté de forme directe.

iii Conventions de transcription: [/] pause silencieuse; [ $\left.{ }^{\circ} \mathrm{h}\right]$ inspiration audible; [ $\left.\uparrow\right]$ intonation ascendante; [:] allongement vocalique ; [()] élision de phonème.

${ }^{\text {iv }}$ On notera au passage que les propos évalués n'ont plus pour sources des experts ou des anonymes, comme dans nos premiers exemples, mais un artiste reconnu, des sportifs de premier plan et deux présidents de la République française.

$\checkmark$ Contrairement aux commentaires, aux chroniques ou aux éditoriaux, où les voix enregistrées sont moins fréquentes, au profit de citations proférées par le journaliste ou d'autres formes de discours représenté. 